\title{
The Microphone Feedback Analogy for Chatter in Machining
}

\author{
Tony Schmitz \\ University of North Carolina at Charlotte, Charlotte, NC 28223, USA \\ Correspondence should be addressed to Tony Schmitz; tony.schmitz@uncc.edu
}

Received 16 March 2015; Accepted 13 May 2015

Academic Editor: Georges Kouroussis

Copyright (C) 2015 Tony Schmitz. This is an open access article distributed under the Creative Commons Attribution License, which permits unrestricted use, distribution, and reproduction in any medium, provided the original work is properly cited.

This paper provides experimental evidence for the analogy between the time-delay feedback in public address systems and chatter in machining. Machining stability theory derived using the Nyquist criterion is applied to predict the squeal frequency in a microphone/speaker setup. Comparisons between predictions and measurements are presented.

\section{Introduction}

Self-excited vibration, or regenerative chatter, in machining has been studied for many years. The research topic has encouraged the application of mathematics, dynamics (linear and nonlinear), controls, heat transfer, tribology, and other disciplines to the field of machining science. In particular, a foundational understanding of regenerative chatter was established by the application of the Nyquist criterion from controls theory to the feedback system generated by machining processes [1]. Many additional publications are available: research in [2] provides a comprehensive review and $[3,4]$ are two available textbooks. The purpose of this paper is to demonstrate the analogy between the squeal frequency experienced when audio feedback causes instability in public address systems $[5,6]$ and the chatter frequency exhibited by machining operations. A simple microphone/speaker setup is described and experimental results are quantitatively and qualitatively compared to machining stability predictions.

\section{Machining Stability Model}

To describe the linear stability analysis, turning is considered here. However, the approach may be extended to milling and other machining processes. When a flexible tool is used to cut away material in the form of a chip, the corresponding cutting force causes time-dependent deflections of the cutting tool. As the vibrating tool removes material, these vibrations are imprinted on the workpiece surface as a wavy profile. Figure 1 depicts an exaggerated view, where the initial impact with the workpiece surface causes the tool to begin vibrating and the oscillations in the normal direction to be copied onto the workpiece [4]. When the workpiece begins its second revolution, the vibrating tool encounters the wavy surface produced during the first revolution. Subsequently, the chip thickness at any instant depends on both the tool deflection at that time and the workpiece surface from the previous revolution. Vibration of the tool causes a variable chip thickness in the sensitive direction, $y$, which yields a variable cutting force since the force is proportional to the chip thickness. See (1), where $F(t)$ is the cutting force, $K_{s}$ is the specific cutting force, $b$ is the chip width, $h_{m}$ is the mean chip thickness, $y(t-\tau)$ is the vibration in the previous revolution, $y(t)$ is the current vibration, and $\tau$ is the time delay (i.e., the time for one revolution). The force is separated into the mean, $F_{m}$, and variable, $F_{v}$, components. Because the cutting force governs the current tool deflection through the structural dynamics, the system exhibits feedback. Consider

$$
\begin{aligned}
F(t) & =K_{s} b\left(h_{m}+y(t-\tau)-y(t)\right) \\
& =K_{s} b h_{m}+K_{s} b(y(t-\tau)-y(t))=F_{m}+F_{v} .
\end{aligned}
$$

Equation (1) can be expressed as a block diagram as shown in Figure 2. The $y$-direction projection of the variable force component (through the force angle, $\beta$ ) excites the structural dynamics in the $y$-direction. The dynamics are described using the frequency response function, or FRF, in that direction. The time-delayed deflection, $y(t-\tau)$, is represented using the exponential function, where $\varepsilon=\tau \omega$ is the phase (in 


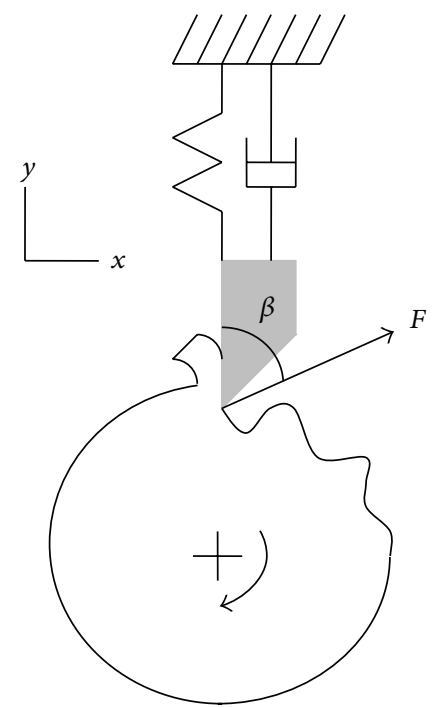

Figure 1: A flexible tool deflects due to the cutting force required to form a chip [4]. These deflections are imprinted on the workpiece surface and cause a variable chip thickness in the next revolution.

rad) between current and previous tool vibrations and $\omega$ is the frequency $(\mathrm{rad} / \mathrm{s})$.

The Nyquist criterion is applied to Figure 2 to identify the limit of stability, where the chip width, $b$, acts as the system gain. The limiting chip width, $b_{\text {lim }}$, is obtained when the open loop transfer function is set equal to -1 as shown in

$$
K_{s} b_{\lim } \operatorname{FRF}\left(1-e^{-j \varepsilon}\right)=-1 .
$$

Equation (2) can be rewritten to relate $b_{\text {lim }}$ to the real (Re) part of the FRF as shown in (3). Additional relationships for the phase and spindle speed are provided in (4) and (5) [4]. Consider

$$
\begin{aligned}
b_{\lim } & =\frac{-1}{2 K_{s} \cos (\beta) \operatorname{Re}[\mathrm{FRF}]}, \\
\frac{f_{c}}{\Omega} & =N+\frac{\varepsilon}{2 \pi}, \\
\varepsilon & =2 \pi-2 \tan ^{-1}\left(\frac{\operatorname{Re}[\mathrm{FRF}]}{\operatorname{Im}[\mathrm{FRF}]}\right) .
\end{aligned}
$$

In (4), $f_{c}$ is the chatter frequency (should it occur), $\Omega$ is the spindle speed, $N$ is the integer number of waves of vibration imprinted on the workpiece surface in one revolution, and $\varepsilon / 2 \pi$ is any additional fraction of a wave. Note that, for units consistency in (4), if $f_{c}$ is expressed in $\mathrm{Hz}, \Omega$ must be specified in rev/s.

In public address systems when a microphone/speaker combination is used to amplify the microphone input, a similar feedback phenomenon is observed. In this case, the speaker output can reenter the microphone after some delay defined by the time for the sound to travel from the speaker to the microphone. This generates a time-delay feedback system

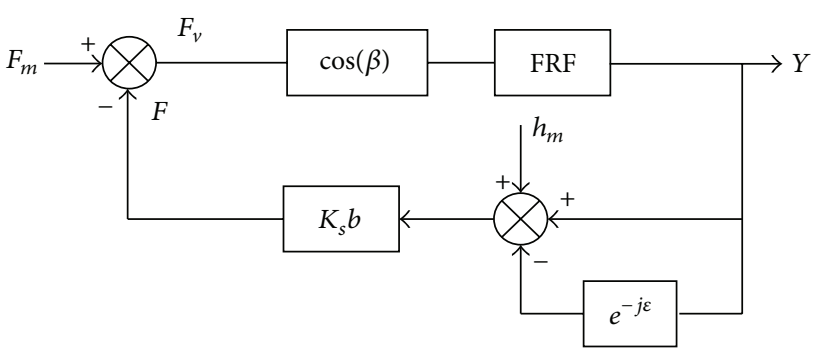

FIGURE 2: Block diagram description of the turning process [1]. The variable component of the cutting force excites the structure's FRF after projection into the $y$-direction. The corresponding frequency domain vibration, $Y$, is summed with the vibration from the previous revolution and mean chip thickness and finally scaled by the product of the specific cutting force and chip width to produce the cutting force.

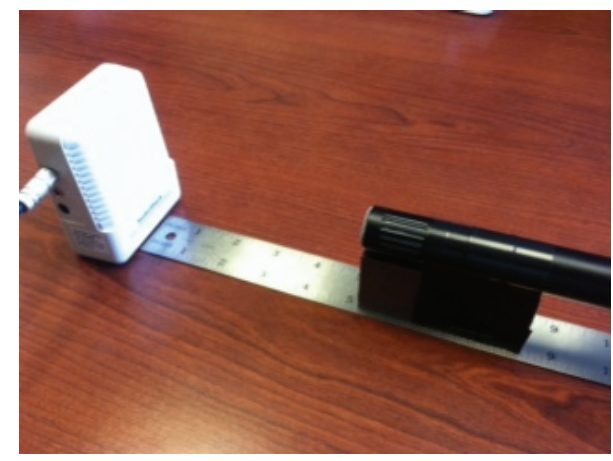

Figure 3: Experimental setup. The speaker is on the left and the unidirectional microphone is on the right. The distance between them is determined using the steel scale.

analogous to the machining case. Relative to the machining case,

(i) the delay is the time for the acoustic wave to travel from the speaker to the microphone (the time for the electrical signal to be generated and transmitted to the speaker is assumed to be constant) and is governed by the distance between the two components;

(ii) the speaker volume takes the place of the chip width; that is, it is the dependent variable that establishes the system stability;

(iii) the FRF is the acoustic/electrical frequency response, rather than the structural dynamics response (in this study, a linear system is assumed although that may not be true in all cases);

(iv) the system gain, independent of the volume, takes the place of $K_{s} \cos (\beta)$.

\section{Experimental Setup}

An experimental setup was constructed to explore the microphone/speaker system instability, which is exhibited as an audible squeal frequency, and demonstrate its similarity to the chatter frequency in machining. 


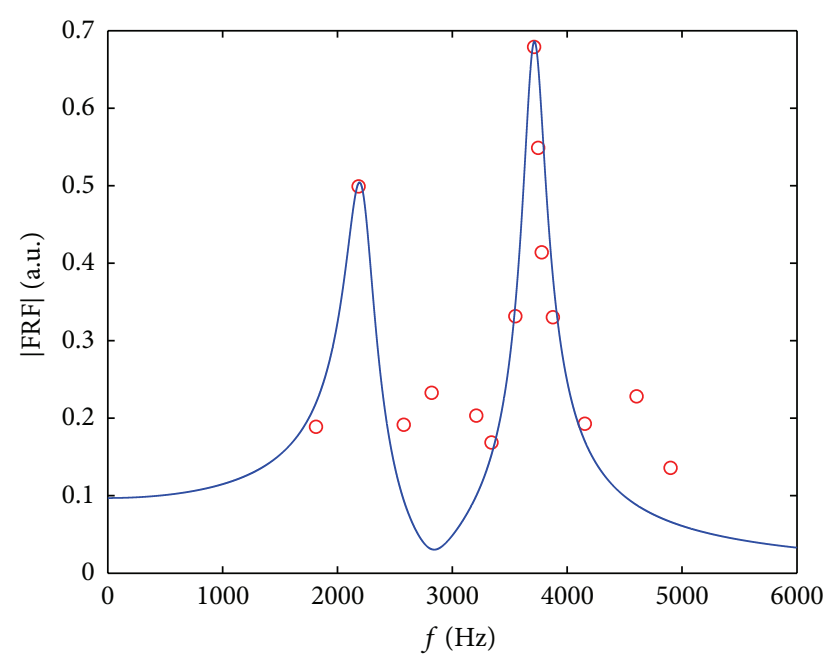

FIGURE 4: Ratio of the speaker output magnitude to the microphone input magnitude in arbitrary units, a.u. (circles). A modal fit (solid line) is also shown.

The setup is pictured in Figure 3, where a Radioshack Mini Amplifier/Speaker and a Switchcraft unidirectional microphone were aligned and the distance between them could be varied continuously. The distance was observed using a steel scale as shown. The speaker volume could also be adjusted to modify the system gain. The speaker volume is the analog to chip width in machining.

\section{Results}

Measurements were performed to identify (1) the system natural frequency(s) and (2) the squeal frequency, $f_{s}$, as a function of the distance between the speaker and microphone, $d$. The natural frequencies were determined by introducing a known audio frequency into the microphone, measuring the speaker output, and then incrementing the frequency over the range of interest. The Fourier transform of both signals was then calculated and the ratio of the speaker output magnitude to the microphone input magnitude at the test frequency was determined (this is the sine sweep approach used in traditional modal testing). This result is displayed in Figure 4; a modal fit is also included, where second-order dynamics were assumed. Although other fits could be considered, only two clear peaks (near $2000 \mathrm{~Hz}$ and $4000 \mathrm{~Hz}$ ) were evident and it was believed that the other points were near the noise floor of the measurements. The modal parameters for the twomode fit are provided in Table 1. For these measurements, the Tone Generator application [7] for the iPhone was used as the frequency source and the Voice Memos application (in the iPhone utilities menu for a second iPhone) was used to record the speaker output $(44.1 \mathrm{kHz}$ sampling rate with no additional digital filtering). The .m4a sound files were then analyzed in MATLAB.

The modal parameters in Table 1 were used to approximately represent the system dynamics as a frequency response function, FRF, and the stability limit and squeal
TABLE 1: Parameters for modal fit to microphone/speaker FRF magnitude.

\begin{tabular}{lccc}
\hline & $\begin{array}{c}\text { Natural } \\
\text { frequency (Hz) }\end{array}$ & $\begin{array}{c}\text { Stiffness } \\
\text { (a.u.) }\end{array}$ & $\begin{array}{c}\text { Viscous } \\
\text { damping ratio }\end{array}$ \\
\hline Mode 1 & 2215 & 17 & 0.06 \\
Mode 2 & 3713 & 26.2 & 0.028 \\
\hline
\end{tabular}

frequency were predicted using (3)-(5). The time delay, $\tau$, was first calculated as the inverse of the predicted spindle speed vector from (4). The time delay was then related to the distance between the speaker and microphone using (6), where $v$ is the velocity of sound in dry air at $20 \mathrm{deg} C$ $(343 \mathrm{~m} / \mathrm{s})$. Consider

$$
d=v \tau \text {. }
$$

Figure 5(a) shows the stability limit as a function of the gain, $K$, and distance. Any $\{d, K\}$ combination above the stability boundaries is predicted to be unstable (i.e., squeal occurs). The vertical axis gain is the chip width in machining but depends on the speaker volume and acoustic cavity (the volume surrounding the speaker and microphone) for the public address system. Because the FRF test results displayed in Figure 4 were obtained with the speaker and microphone separated (to separate the source and response measurements), they do not include the inherent acoustic cavity effects. Therefore, the gain units are not provided and only qualitative comparisons between the speaker volume (gain) and stability can be completed; when applying (3), the product $K_{s} \cos (\beta)$ was assumed to be unity.

Figure 5(b) shows the chatter frequency as a function of the distance between the speaker and microphone. In this case, the squeal frequency could be measured using the Voice Memos application so a direct comparison is possible. The chatter frequency prediction from the machining stability analysis is shown as the solid line, while the squeal frequency measurements are plotted as circles. An example spectrum for the $d=25 \mathrm{~mm}$ test is displayed in Figure 6. It is observed that a single frequency (at $5330 \mathrm{~Hz}$ ) is present.

The squeal frequency results in Figure 5 show good quantitative agreement with the machining analysis chatter frequencies in all cases except for the smallest distance between the speaker and microphone ( $d=15.9 \mathrm{~mm}$ point). It is proposed that this unstable result is due to acoustic cavity effects and is outside the scope of this study. To provide a qualitative stability comparison, the speaker volume was varied from high to low and the regions of squeal (unstable) and no squeal (stable) were recorded. The results for three volume, or gain, values are presented in Figure 7. Because the cavity gain was not measured, the vertical locations of the three gains (i.e., the horizontal lines in Figure 7) were selected to approximately match the stability boundary in Figure 7 . This is equivalent to applying the machining stability predictions to a system, where $K_{s}$ and $\beta$ are unknown. In this case it is possible to identify preferred spindle speeds (horizontal axis), but not limiting depths of cut (vertical axis). Qualitative assessment of stability is possible, but vertical scaling of the chip width is not. Figure 7 also depicts the disagreement 


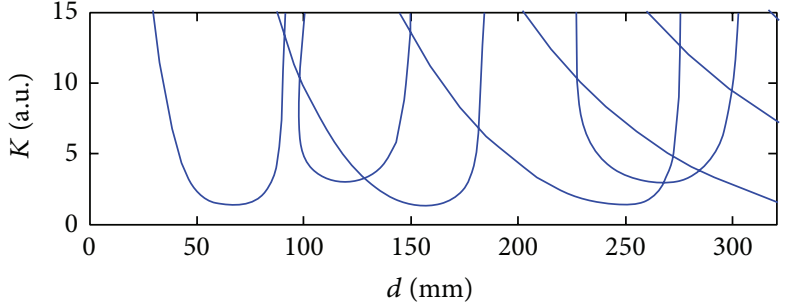

(a)

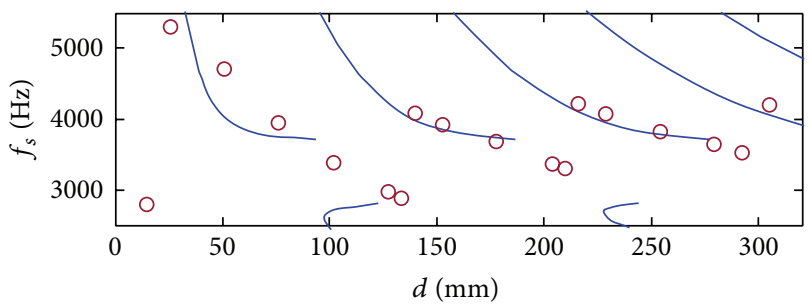

(b)

FIGURE 5: (a) Stability prediction as a function of distance between the speaker and microphone, $d$, and the audio system gain, $K$ (arbitrary units, a.u.). The solid lines indicate the stability boundaries (unstable above). (b) Squeal frequency, $f_{s}$, prediction as a function of $d$ (solid line). Experimental results are shown as open circles.

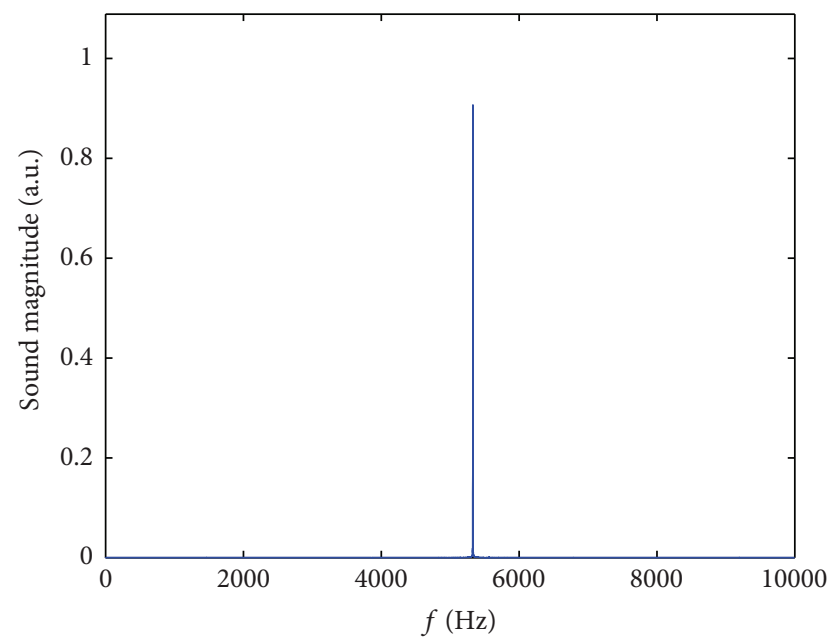

FIGURE 6: Spectrum for the $d=25 \mathrm{~mm}$ test is from Figure 5(b). A single frequency at $5330 \mathrm{~Hz}$ is observed.

between prediction and measurement at low $d$ values. Again, it is assumed that the system dynamics change when the cavity is sufficiently small.

\section{Discussion}

While the bottom panel of Figure 5 provides clear evidence that the squeal frequency produced by time-delay feedback in the speaker/microphone setup provides an analogy to the chatter frequency in machining, a more compelling expression of the behavior is established by video (with audio support) from a continuous scan of the distance between the speaker and microphone. It is seen and heard that the squeal frequency periodically rises and falls as the distance is varied; the video is available online [8].

In addition, video is provided that shows both a forward (microphone moving toward the speaker) and reverse direction sweep [8]. As displayed in Figure 8, it is observed that the dramatic shifts in squeal frequency (bifurcations) do not occur at the same spatial locations between the two directions. In fact, the separation between the forward and reverse direction bifurcations depends on the speed of the microphone as it moves relative to the speaker; higher speeds

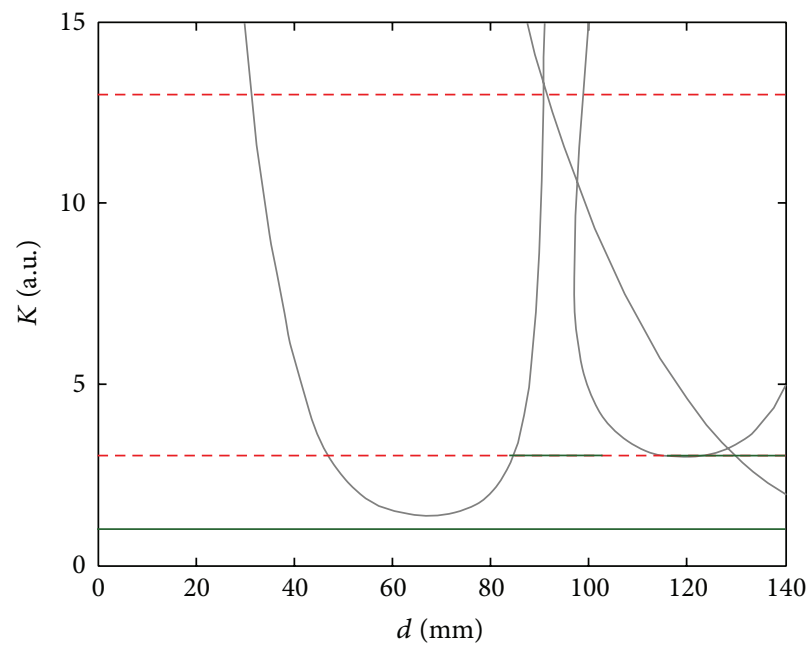

FIGURE 7: Stability comparison at three speaker volume (gain) values. The highest gain (top line) exhibited squeal at all locations. The dotted line indicates squeal/instability. This gain provided the squeal frequencies for Figure 5. A lower volume produced squeal only at particular distances (middle line). Stable (no squeal) distances are identified by the solid line. Below a threshold volume value, no squeal occurs (bottom line).

give a larger separation. This is indicative of nonlinear behavior. While the goal of this study was to simply demonstrate the similarity between the machining and public address systems due to the presence of time-delay feedback, additional studies could be completed to further explore the nonlinear behavior inherent to each $[9,10]$.

\section{Conclusions}

It was demonstrated that a direct analogy exists between audio feedback in public address systems and chatter in machining; both are time-delay feedback systems with stability that depends on the system gain. The stability limit and chatter frequency equations for machining derived using the Nyquist criterion were applied to predict the squeal frequency for a microphone/speaker setup. It was shown that the squeal frequency could be predicted and that qualitative agreement in the stability predictions with variable gain 


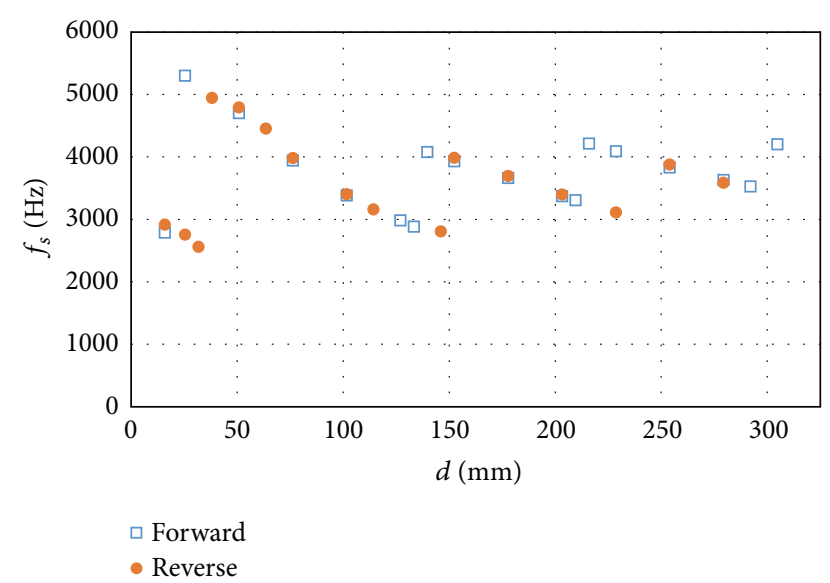

FIGURE 8: A discrepancy between the shifts in squeal frequency with distance is observed between the forward (microphone moving toward the speaker) and reverse paths. This indicates nonlinear behavior for the speaker-microphone system.

(speaker volume) and distance between the speaker and microphone were obtained.

\section{Conflict of Interests}

The author declares that there is no conflict of interests regarding the publication of this paper.

\section{Acknowledgment}

The author gratefully acknowledges helpful discussions with Dr. M. Davies, University of North Carolina at Charlotte.

\section{References}

[1] J. Tlusty, Manufacturing Processes and Equipment, PrenticeHall, Upper Saddle River, NJ, USA, 2000.

[2] Y. Altintas and M. Weck, "Chatter stability of metal cutting and grinding," CIRP Annals-Manufacturing Technology, vol. 53, no. 2, pp. 619-642, 2004.

[3] Y. Altintas, Manufacturing Automation: Metal Cutting Mechanics, Machine Tool Vibrations, and CNC Design, Cambridge University Press, Cambridge, UK, 2000.

[4] T. Schmitz and K. S. Smith, Machining Dynamics: Frequency Response to Improved Productivity, Springer, New York, NY, USA, 2009.

[5] M. R. Schroeder, "Improvement of acoustic-feedback stability by frequency shifting," The Journal of the Acoustical Society of America, vol. 36, no. 9, pp. 1718-1724, 1964.

[6] M. Kidd and G. Stépán, "Delayed control of an elastic beam," International Journal of Dynamics and Control, vol. 2, no. 1, pp. 68-76, 2014.

[7] http://www.lifegrit.com/.

[8] http://coefs.uncc.edu/tschmit4/videos/.

[9] F. C. Moon and T. Kalmár-Nagy, "Nonlinear models for complex dynamics in cutting materials," Philosophical Transactions of the Royal Society A, vol. 359, no. 1781, pp. 695-711, 2001.
[10] K. Gu and S.-I. Niculescu, "Survey on recent results in the stability and control of time-delay systems," Journal of Dynamic Systems, Measurement and Control, vol. 125, no. 2, pp. 158-165, 2003. 

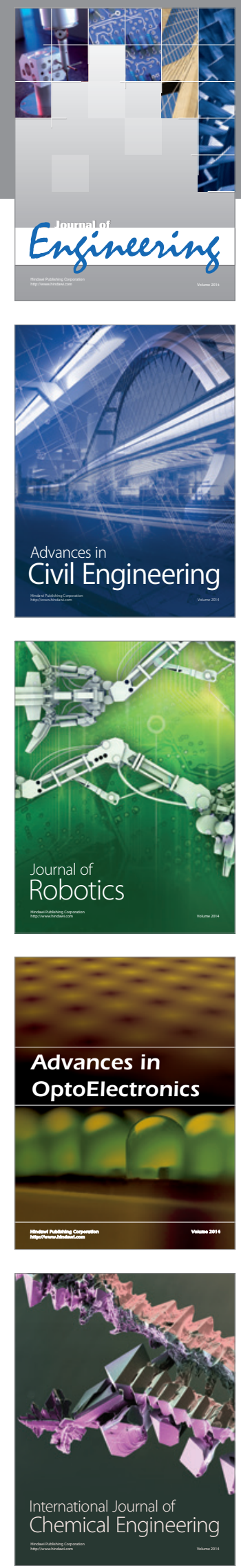

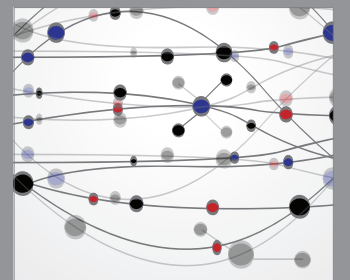

The Scientific World Journal
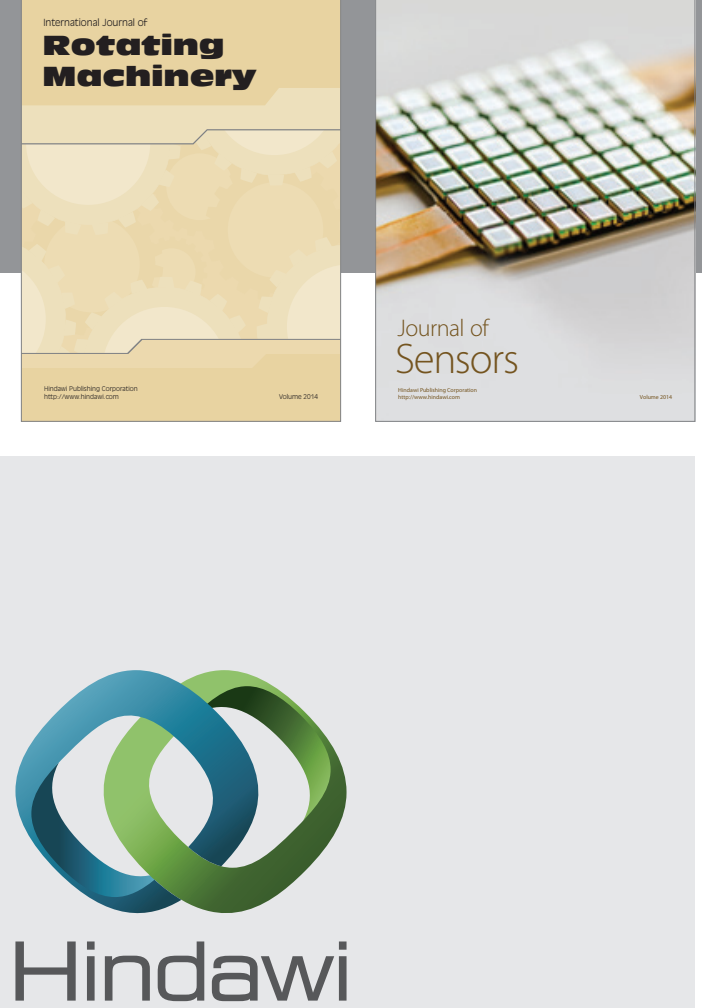

Submit your manuscripts at http://www.hindawi.com
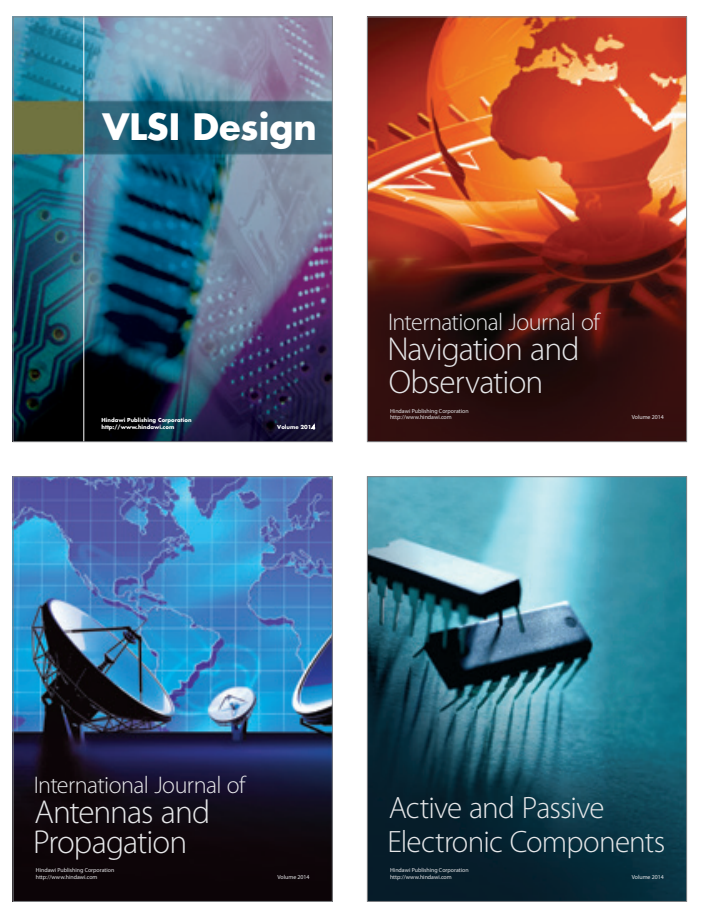
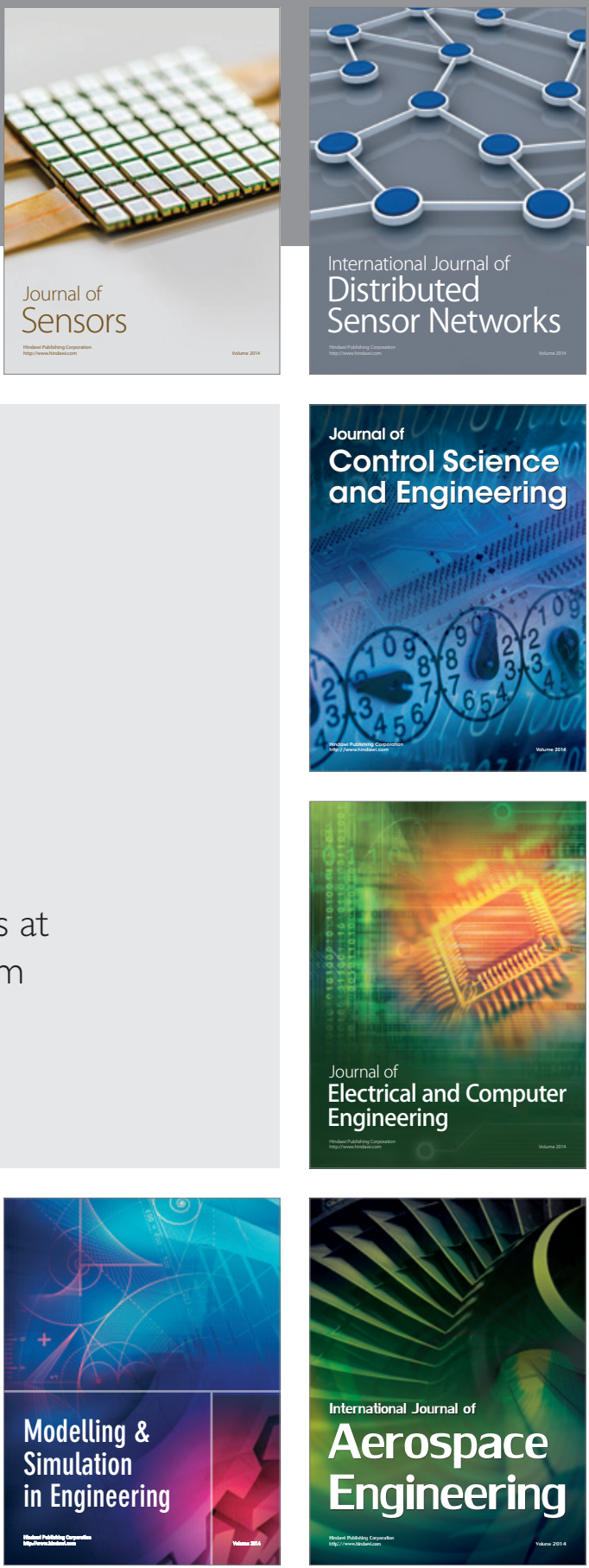

Journal of

Control Science

and Engineering
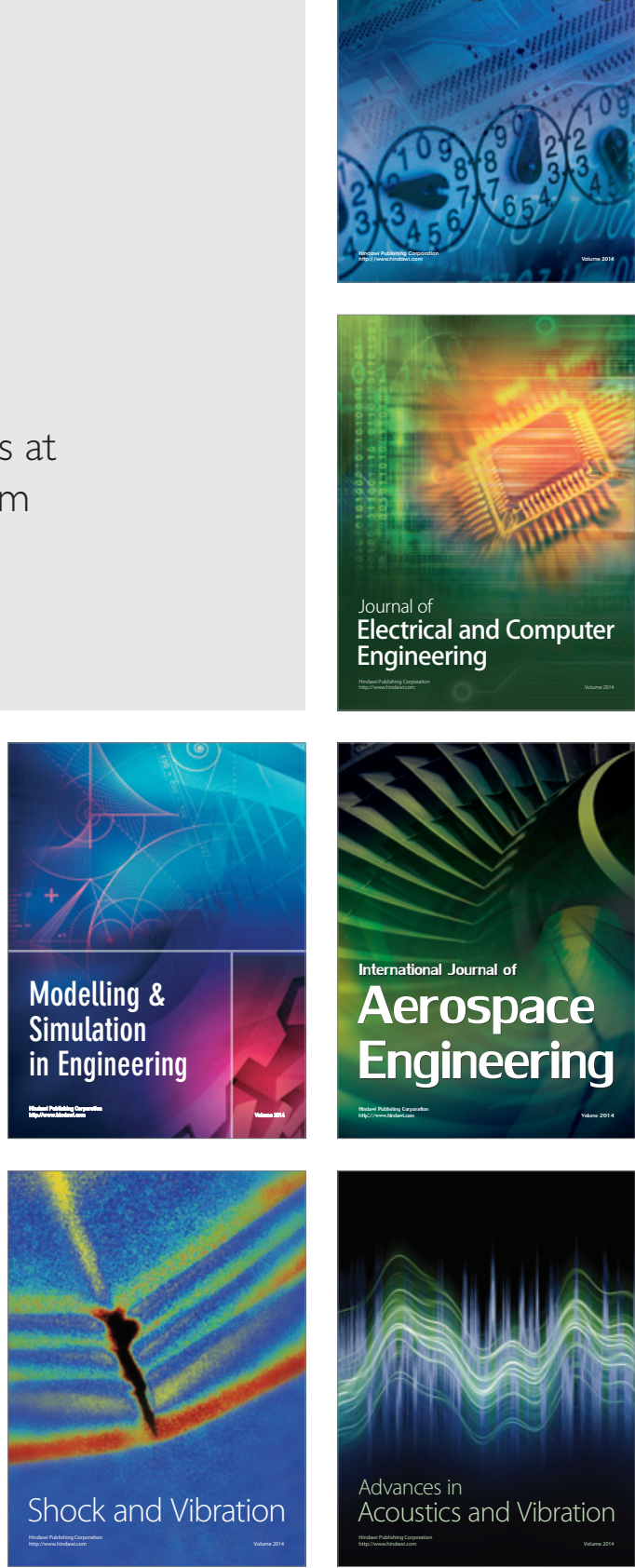